\title{
Women's attitudes towards heavy menstrual bleeding, and their impact on quality of life
}

This article was published in the following Dove Press journal:

Open Access Journal of Contraception

23 April 2013

Number of times this article has been viewed

\author{
Johannes Bitzer' \\ Marco Serrani ${ }^{2}$ \\ Annalena Lahav² \\ 'Universitats-Frauenspital Basel, \\ Basel, Switzerland; ${ }^{2}$ Bayer \\ HealthCare AG, Berlin, Germany
}

Correspondence: Johannes Bitzer Universitätsspital Basel, Frauenklinik, Chefarzt/Vorsteher, Spitalstrasse 2I, $\mathrm{CH}-403 \mathrm{I}$, Basel, Switzerland

Tel +4I 6I 2659099

Fax +4| 6I $2659 \mid 99$

Email johannes.bitzer@usb.ch
Abstract: The aim of this study was to gain an in-depth understanding of women's current knowledge, attitudes, and beliefs around heavy menstrual bleeding (HMB) and gain a further understanding of how HMB affects women's lives, while identifying current misconceptions and gaps in the knowledge. A total of 6179 women, 18-55 years old, currently using, or open to using, hormonal contraception at the time of the study, were recruited from 15 countries. All participants completed a 52 question online survey designed to assess the impact of HMB on quality of life, as well as current levels of awareness and knowledge of this condition. Of the women who perceived themselves as having above average menstrual flow, a significantly greater proportion identified their menstrual bleeding as problematic compared to those women who perceived themselves as having below average menstrual flow. Further questioning indicated that the impact of diagnosed or perceived HMB stretched across multiple aspects of women's lives, including social life, relationships, and work, with significantly greater proportions of women reporting negative effects in these domains in the above average menstrual flow subgroup relative to the below average menstrual flow subgroup $(P<0.05)$. In addition, awareness and understanding of HMB within the survey population were found to be poor, with $48 \%$ of participants claiming that they were "not at all" or "not very" knowledgeable regarding HMB and $39 \%$ of those diagnosed with the condition believed that no treatment was available to them. This study confirms previous findings indicating that HMB has a profound negative effect on many aspects of women's lives and shows that, despite its common occurrence, gaps in women's current knowledge and understanding of HMB remain.

Keywords: menorrhagia, quality of life, (QoL), menstrual flow

\section{Introduction}

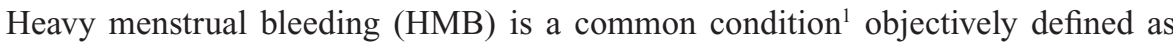
menstrual blood loss (MBL) of $\geq 80 \mathrm{~mL}$ per cycle. ${ }^{2}$ This threshold value of MBL was set due to the pathologic consequences of MBL of this volume, as this is associated with a high frequency of iron deficiency. ${ }^{2}$ Considering the time and expense that it takes to accurately measure MBL volume, objective definitions such as this are often deemed impractical for use in daily clinical practice. The objective definitions also fail to take into account women's wellbeing, in addition to any impact that HMB may have upon their quality of life (QoL). This is an important factor to consider, given that HMB has been shown to cause considerable morbidity. ${ }^{1,3,4}$

There have been many studies conducted to assess the prevalence of $\mathrm{HMB},{ }^{1}$ and estimates based on their findings vary considerably. One of the causal factors attributed to the wide range of prevalence findings is the discrepancy in the definition of HMB. 
When measured quantitatively, HMB prevalence has been found to range from $9 \%$ to $14 \%,{ }^{1}$ yet when measured qualitatively, it has been found to be as high as $52 \% .^{5}$ Despite these differences, HMB is known to be a widespread medical condition, being one of the most common ailments presented to obstetricians and gynecologists by women. ${ }^{6}$

Due to the impracticality of measuring HMB objectively in daily clinical practice, the most recent National Institute of Health and Clinical Excellence guidelines now subjectively define HMB as "excessive blood loss which interferes with the woman's physical, emotional, social and material quality of life."' With diagnostic procedures being brought in line with this definition, medical consultations will become increasingly focused on women's perceptions of menstrual flow and the effect it has on QoL. These perceptions can easily be established via informed patient questioning as part of the diagnostic process used by health care professionals (HCPs). This makes subjective definitions more practical for use in daily clinical practice.

Women's perceptions of what is "normal" with regards to menstruation depend largely upon their understanding of menstruation and what they perceive its function to be. ${ }^{8}$ It is important that HCPs understand each woman's point of view, as this could help inform the questioning carried out as part of the medical consultation process. The concept of menstruation has been found to vary greatly across different cultures, as well as throughout history. ${ }^{8}$ The varying ideas often do not align with what is medically proven. The idea that menstruation is natural and therefore "beneficial" dates back to the time of Hippocrates when the reputed "father of modern medicine" described menstruation as "purging women of bad humours." "This view of menstruation as a monthly cleanser has given rise to the idea that when women experience excessive MBL, it is medically beneficial. ${ }^{8}$ Menstrual blood itself has also been described as detrimental and has even been depicted as a "deadly poison" in the famous book of natural history, written in approximately 77 AD. ${ }^{8}$ These views largely inspired the use of bloodletting in medical practice, which up until the beginning of the twentieth century, was commonly used to treat many medical conditions by even the most eminent medical authorities. ${ }^{8}$

Throughout history, women have often been made to feel that menstruation is a topic that should not be discussed openly. ${ }^{8}$ In the US, even as recently as the 1970 s and 80 s, women were often exposed to messages indicating that menstruation is something "messy" and "shameful" that they should try to conceal. ${ }^{8}$ As a cyclical event, the medical risk attributed to menstruation is often low. ${ }^{8}$ This means that dysfunctional uterine bleeding conditions, such as HMB, may go undiagnosed and untreated, leading to unnecessary suffering in women burdened by this type of condition.

Variation in the questions posed by HCPs during patient consultations may help to correctly diagnose and treat HMB, thereby meeting the patient's needs. A recent study by Matteson et al investigated whether the questions used by HCPs to diagnose HMB adequately covered the aspects of women's lives that were impacted by this condition. ${ }^{3}$ This was done in conjunction with qualitative research to explore the effects that women felt HMB had on their lives. The results identified a number of areas not adequately covered by standard HCP questioning, and recommended additional areas of questioning on which to focus. ${ }^{3}$ These areas included: time taken to soak through a large sanitary pad, frequency of restroom visits to check or change sanitary protection, and frequency of passing blood clots. Despite providing useful information, this study investigated a small $(n=25)$, nonrandom sample, which limited the extent to which the study population could be considered representative and, therefore, the ease with which the findings could be generalized. ${ }^{3}$

Other studies that have assessed the effect of HMB on QoL ${ }^{4,10}$ have found similar results in terms of the areas of women's lives that are affected. These areas include social life, relationships, and daily activities. Despite being informative, few studies to date have looked at these trends on a global scale or vigorously assessed women's knowledge surrounding HMB. In light of this, more comprehensive research is needed in order to gain further understanding of how HMB affects women's lives and where the knowledge gaps and misconceptions lie.

\section{Materials and methods}

The objective of this study was to gain an in-depth understanding of women's current knowledge, attitudes, and beliefs pertaining to HMB. The study population was recruited via an email generated from the database of an international survey panel provider. The email invitation contained a link to an online prescreening survey, which determined the eligibility of women interested in participating in the study and ensured that they fulfilled the demographic criteria for inclusion. Participants were required to be women, aged 18-55 years of age, who had not undergone a hysterectomy and were not currently taking hormone replacement therapy. In addition, only women currently using, or open to using, hormonal contraception at the time the study was conducted were eligible to participate. If these criteria were fulfilled, the 
respondent was directed to the main online survey. Women who did not fit these criteria did not complete the second stage of the survey process. Female panel members across 15 countries were invited to take part.

The main questionnaire was a 52 item online survey in the local language of the respondents, which took approximately 25 minutes to complete. Women were provided with a small incentive to participate, in the form of reward points, which constituted part of the incentive scheme operated by the survey panel provider used. Points can be collected by those who complete surveys for the panel, and then exchanged for gifts of varying value depending on the number of points collected.

Two of the 52 questions were open ended, with the rest being closed, fixed answer questions. Answers to open ended questions were subsequently coded and categorized during data processing. All surveys were completed in December 2010.

Established data from the peer-reviewed literature were used as the basis for development of the survey questions. Questions were designed to cover the following topic areas within HMB:

- impact of HMB on QoL;

- current awareness and knowledge of HMB;

- and diagnosis and interaction with HCPs in relation to HMB.

Specific survey questions were used "up front" in order to identify women who had been diagnosed with HMB, as well as to split the survey population according to whether they perceived their menstrual flow to be above or below average. This allowed statistical comparison of patient subgroups in terms of knowledge, attitudes, and beliefs around HMB for subsequent survey questions. One of the main interests of the study was the effect of HMB on different aspects of QoL and daily activities. Items used in the questionnaire to assess the QoL consequences of HMB were developed specifically for the purposes of the study.

Following completion of the online survey, any incomplete or nonsensical survey answers were removed from the data set to reduce error variation. Following this, 6179 study participants remained, distributed across a total of 15 countries (Table 1). Prior to statistical analysis, the raw data were weighted according to female population size within the study participants' respective countries (Bayer HealthCare, data on file, 2010). This was done to ensure that results from each country did not disproportionately influence the global trends due to variations in their respective female population size (Table 1).
Table I Breakdown of study participants by country, and country distribution after weighting by female population size (Bayer, data on file, 2010)

\begin{tabular}{lll}
\hline Study country & Sample size $(\mathbf{n})$ & Weighting factor \\
\hline Australia & 394 & 0.298098 \\
Argentina & 391 & 0.530043 \\
Brazil & 374 & 2.963426 \\
Canada & 386 & 0.478428 \\
France & 417 & 0.751947 \\
Germany & 403 & 1.048747 \\
Italy & 397 & 0.786888 \\
Mexico & 376 & 1.577887 \\
Poland & 375 & 0.56961 \\
South Korea & 387 & 0.748054 \\
Spain & 379 & 0.674617 \\
Sweden & 388 & 0.117305 \\
Thailand & 349 & 1.15625 \\
UK & 387 & 0.831413 \\
USA & 776 & 2.075822 \\
\hline
\end{tabular}

Statistical analyses were conducted using two sample $t$-tests to test for significant differences between arithmetic means, and Z-tests to test for significant differences between column proportions. All tests were two-tailed and conducted with a $95 \%$ confidence interval to ensure significance.

Sliding scales of multiple choice options were given to many of the fixed answer questions, for example: "strongly disagree", "somewhat disagree", "somewhat agree", "strongly agree". The percentages given in the results section reflect the proportion of women selecting one of the top two answers to each of these questions (eg, "strongly agree" and "somewhat agree").

\section{Results}

A total of 6179 women met the eligibility criteria for the study and went on to fully complete the 52 question online survey. From the "up front" questioning, 887 of these women stated that they had been diagnosed with HMB, creating the HMB diagnosed (HMBd) subgroup. A total of 1627 women perceived their menstrual flow to be above average, creating a perceived above average menstrual flow (AAMF) subgroup. There was some overlap between subgroups, meaning that 499 women were included in both groups. Herein, we focus mainly on the AAMF subgroup, as this group captures women's subjective perceptions, regardless of a formal diagnosis of HMB. The subjective nature of this measure is in line with recent National Institute of Health and Clinical Excellence guidelines. ${ }^{7}$

Although the AAMF subgroup provides informative subjective data, in terms of assessing total prevalence of $\mathrm{HMB}$, we need to consider both the HMBd and AAMF 
subgroups together. In terms of this top line prevalence, $36 \%$ of women surveyed stated that they had either been diagnosed with HMB or felt that their menstrual flow was above average (weighted by female population size in the respective countries). This is within the range of previous estimates for the prevalence of $\mathrm{HMB}^{1}$ and reinforces the findings of previous research ${ }^{5,11,12}$ showing $\mathrm{HMB}$ to be a common condition.

\section{Impact of HMB on QoL}

More women in the AAMF subgroup identified their menstrual cycle as being an inconvenience and disruption to their lives compared to those women with perceived below average menstrual flow (BAMF). In the AAMF subgroup, 83\% of women stated that HMB impacted upon their daily activities and $75 \%$ stated that their period was a major inconvenience to their lives (Figures 1 and 2, respectively); both of these percentages were significantly higher $(P<0.05)$ than those for the BAMF subgroup.

Further questioning showed that the impact of diagnosed or perceived HMB stretched across multiple aspects of women's lives, including social life, relationships, and work, as detailed in the following sections of this paper.

\section{Social life}

In the AAMF subgroup, $68 \%$ of women stated that they tend not to participate in social activities when their period is heavy (Figure 2) and 91\% of women felt that it impacted their ability to perform sport and fitness activities. A high proportion of women in this subgroup (91\%) also felt that their heaviest menstrual flow days impacted on the type and color of clothes they wore, with $82 \%$ of these women commonly wearing certain underwear when they are on their period. As well as the physical implications, $75 \%$ of this subgroup said that they feel less attractive and less confident during their period (Figure 1). Just less than half of these women (47\%) also stated that, on occasion, HMB even prevented them from playing with their children (Figure 2). All reported values were significantly higher in the AAMF subgroup versus the BAMF subgroup $(P<0.05)$.

\section{Relationships}

Significantly more women in the AAMF subgroup stated that their periods impacted on their relationships compared to women in the BAMF subgroup $(P<0.05)$. A large proportion of these women (84\%) stated that they avoided sexual activity when their period was heavy (Figure 1) and $82 \%$ stated that

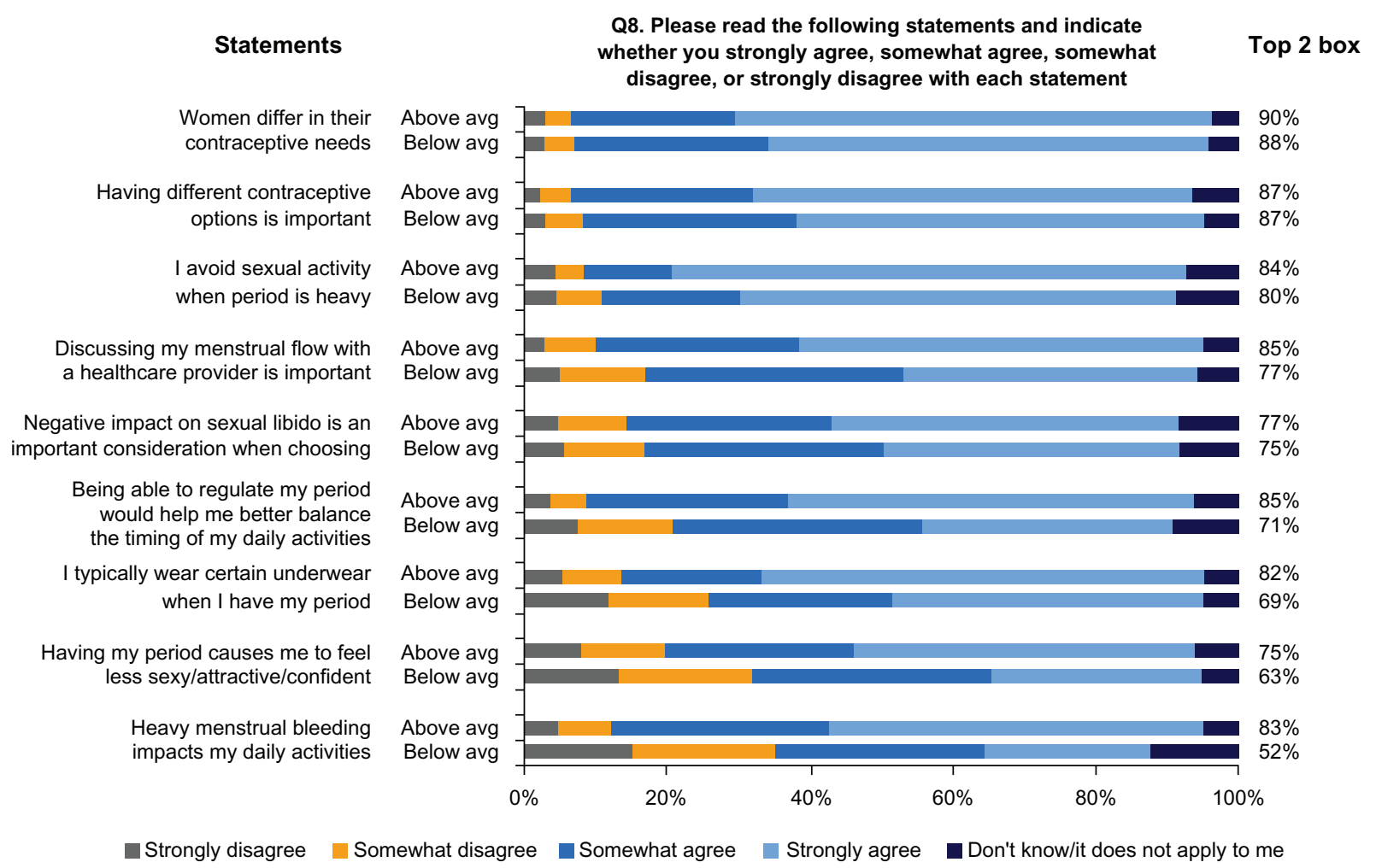

Figure I Feelings on the implications of menstrual flow.

Notes: $n=1627$ with AAMF; $n=4227$ with BAMF.

Abbreviations: AAMF, above average menstrual flow; avg, average; BAMF, below average menstrual flow. 


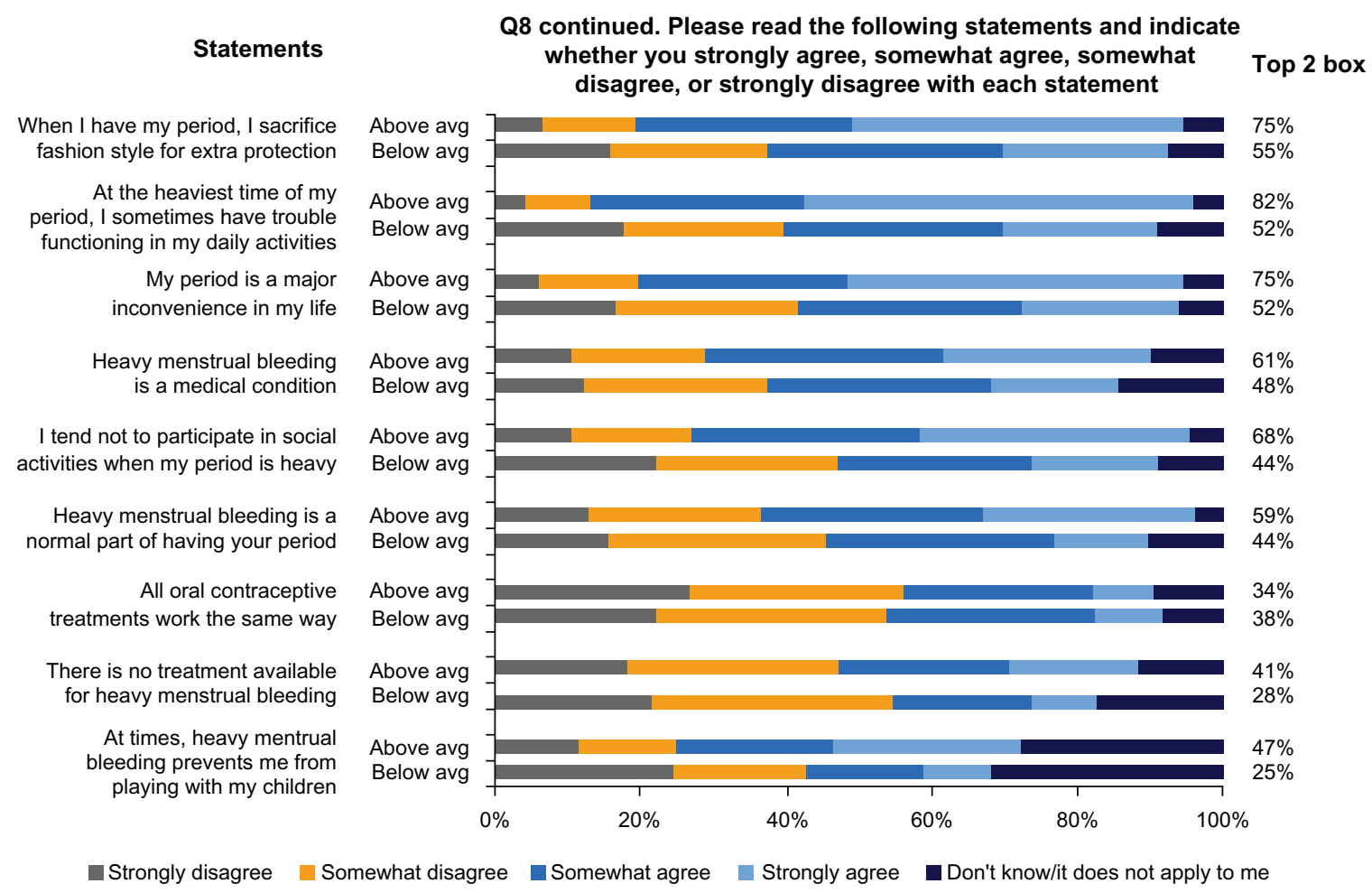

Figure 2 Feelings on the implications of menstrual flow.

Notes: $\mathrm{n}=1627$ with AAMF; $\mathrm{n}=4227$ with BAMF.

Abbreviations: AAMF, above average menstrual flow; avg, average; BAMF below average menstrual flow.

their heaviest flow days impacted on their relationships with their partners.

\section{Work}

This research provides further evidence of the impact HMB may have on productivity. Results showed that $68 \%$ of women in the AAMF subgroup felt that their heaviest flow days impacted on their attendance at work and/or school. The majority of women $(80 \%)$ in the AAMF subgroup also stated that their heaviest flow days affected their performance at work and/or school. The proportion of women in the AAMF subgroup who felt that HMB negatively affected their work was significantly higher than that reported by women in the BAMF subgroup $(P<0.05)$. Outside of employment, heavy flow days were also shown to impact on daily tasks at home, with $73 \%$ of women in the AAMF subgroup stating that their heaviest flow days impacted on their usual housekeeping tasks.

\section{Management of HMB}

Previous research has demonstrated that women affected by HMB often adopt lifestyle changes in order to manage their condition. ${ }^{3}$ When women in the HMBd subgroup were questioned about this, answers fell into several categories (Figure 3). In terms of physical methods of management, $84 \%$ said that they wore certain underwear during their periods and $83 \%$ said they wore specific clothes. A large percentage of women (74\%) stated that they avoided sitting for long periods of time and $77 \%$ regularly used multiple sanitary items simultaneously. In terms of social life, $63 \%$ stated that they planned events around their menstrual cycles and $69 \%$ went as far as skipping social events altogether. With regards to relationships, $87 \%$ of women in this subgroup said that they avoided sexual activity during their period in order to manage their HMB.

In terms of medical management, $62 \%$ of the $\mathrm{HMBd}$ subgroup stated that they used prescribed and/or selfadministered over the counter treatment in order to treat their HMB or its associated symptoms.

\section{Awareness and knowledge of $\mathrm{HMB}$}

Despite being a common condition that impacts many aspects of women's lives, awareness of HMB amongst the survey participants was found to be low. When the global study population was asked if they had heard of HMB, $34 \%$ of them answered "no" or "don't know". Following on from this, $48 \%$ claimed that they were "not at all" or "not very" knowledgeable about HMB. Even within the 


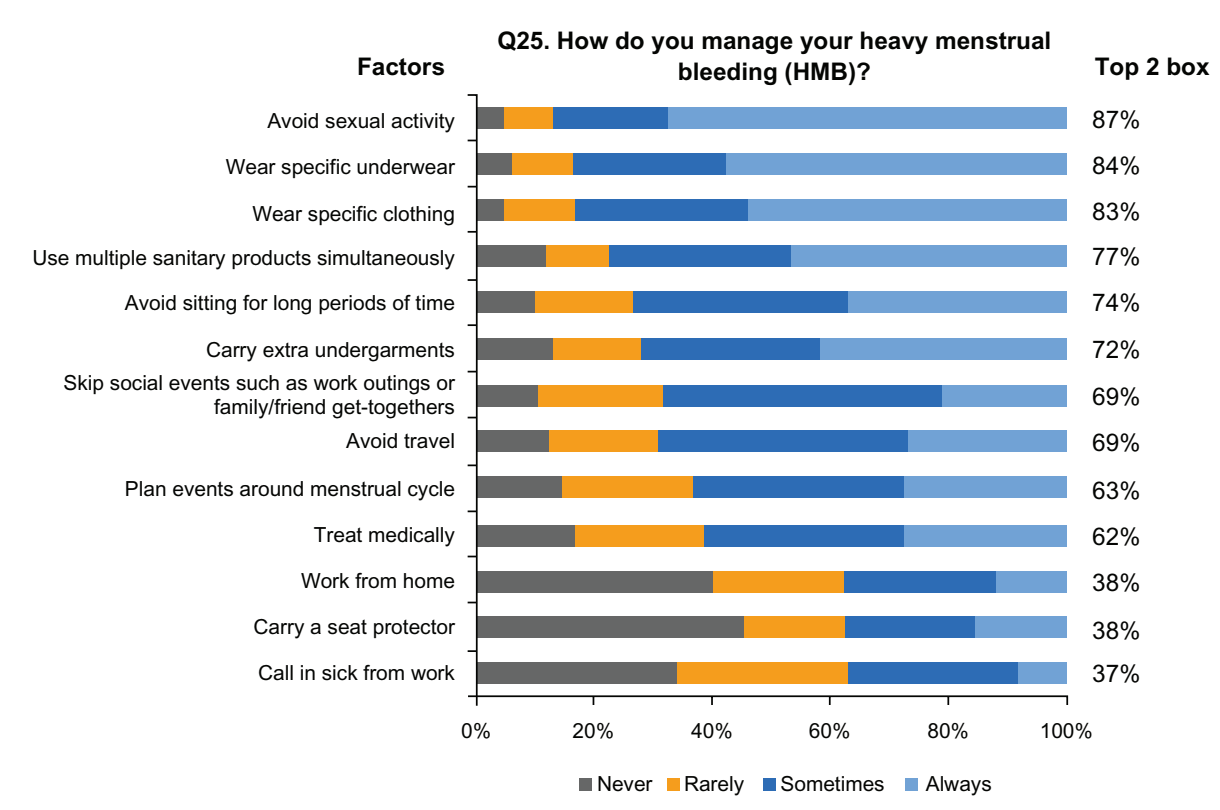

Figure 3 How women diagnosed with HMB manage their condition.

Note: Global total of $n=887$ woman who have been diagnosed with HMB.

AAMF subgroup, 59\% accepted HMB to be a normal part of menstruation (Figure 2). The trend of being unsure with regards to what is normal continued into the global study population where, when asked what they thought the average volume of MBL was during a normal menstrual cycle, $48 \%$ of participants overestimated (range of answers given). For the purpose of calculation, 'normal' MBL was taken to be 35-45 mL ( \pm standard deviation 25-30 mL). ${ }^{2,13}$

Even within the HMBd subgroup, understanding of HMB and available treatment options was found to be lacking. Thirty-five percent of the women in this subgroup stated that they knew nothing about the condition, or that their knowledge was poor. Furthermore, 39\% of those women in the HMBd subgroup believed that there was no treatment available to them, as did $41 \%$ in the AAMF subgroup (Figure 2). The confusion as to what is normal is further exemplified by the fact that $43 \%$ of women in the $\mathrm{HMBd}$ subgroup did not acknowledge an above average menstrual flow when asked to comment on their own MBL, though this may be due to treatment that members of this subgroup were receiving for $\mathrm{HMB}$.

\section{Interaction with HCPs}

The apparent lack of knowledge surrounding HMB and the available treatment options may be one of the causal factors as to why many women affected by HMB do not seek medical advice. In this study, 35\% of women in the AAMF subgroup had not discussed HMB with their HCP. When asked why,
$18 \%$ stated that they were too embarrassed and 19\% stated they thought there was nothing that the HCP could do. Even amongst the HMBd subgroup, $33 \%$ of women had taken more than a year to seek medical advice.

\section{Discussion}

This study supports the findings of previous research ${ }^{1,3,4}$ showing HMB to be a common problem which can impact on many aspects of a women's life, including social life, relationships, and work. Such results reinforce the need to consider women's perceptions of MBL and the subsequent impact on their QoL. The results from this study show that significantly more women in the AAMF subgroup identified their menstrual bleeding as problematic in comparison to those women in the BAMF subgroup. Despite the associated morbidity implied by these findings, many of the women in the AAMF subgroup may not undergo an objective assessment or meet objective requirements for a clinical diagnosis of HMB. Subjective information could be used alongside objective measures in order to strengthen the diagnostic procedures used in daily clinical practice and bring them in line with recent National Institute of Health and Clinical Excellence guidelines. ${ }^{7}$ In order to improve the way in which patients are diagnosed with menstrual bleeding disorders, HCPs may need to be given further guidance regarding the definition of HMB, as this will influence their questioning and practice during clinical consultations. 
Despite it being a common condition, awareness and understanding of HMB within our survey population was found to be poor. Many had not heard of HMB, and nearly half had no knowledge, or very little knowledge, about the condition. This trend extended into the HMBd subgroup, suggesting a possible breakdown of communication between the HCP and patient in terms of disseminating information post-diagnosis. As well as a general knowledge gap, 39\% of women in the HMBd subgroup were not aware of any treatment options for HMB, despite the fact that there are many. ${ }^{714-20}$ The most commonly used medical treatments available to patients with no underlying organic pathology include the levonorgestrel releasing intrauterine system, non-steroidal anti-inflammatory drugs, antifibrinolytics, cyclical progestins, and the modified testosterone derivative, danazol. ${ }^{14-18}$ Combined oral contraceptives have also been found to reduce MBL by $35 \%-43 \%$ and, as a result, are often used off label. ${ }^{7,19,20}$ Recent advances in this category have seen the introduction of an oral contraceptive that combines estradiol and dienogest, which has become the only combined oral contraceptive indicated for the treatment of $\mathrm{HMB}^{21}$ with a rapid MBL reduction (reaching $88 \%$ after 6 months of treatment). ${ }^{22}$

The results from this study suggest that there is a need to raise awareness of HMB and its treatment options amongst women, as well as provide necessary guidance and resources to HCPs in order to enable them to disseminate appropriate information to patients with this condition.

During this study, women's perceptions of what is normal were also found to vary amongst our survey participants. Many women in the HMBd and AAMF subgroups accepted that HMB was just a normal part of having a period. The acceptance of above average bleeding by many women in this study may be a reflection of what these women perceive the purpose of menstruation to be (eg, a monthly purging or cleansing of bodily fluids). Although further research is needed, the data from this study support the hypothesis that an increased emphasis needs to be placed on exploring women's perceptions of menstruation in a clinical diagnosis or counseling setting. This would help to identify what women perceive as 'normal' and provide an opportunity to gain a better understanding of how HMB affects their QoL. This, in turn, could assist with the accurate diagnosis and treatment of HMB.

Research from this study and other similar studies could be used to help direct the questioning HCPs employ during diagnostic interviews, providing insight into the areas of women's lives most affected by HMB, and providing HCPs with new tools to aid diagnosis. In order to strengthen patient identification further, HCPs may also need to receive more extensive guidance regarding other common presenting symptoms of HMB, such as anemia. ${ }^{1}$ This could help HCPs to ask informed questions and identify an increasing number of female patients for whom HMB is the underlying cause of their presenting anemia.

As far as we are aware, this is the largest $(n=6179)$ international study of its kind to provide an insight into women's knowledge, attitudes, and beliefs around HMB, the results for which encompass a wide range of demographics. In terms of study limitations, questions in this study were largely closed, restricting the responses given by study participants. These data should therefore be used in conjunction with research that is more qualitative in nature, in order to build a more comprehensive picture of women's attitudes and beliefs around HMB. Returning to the eligibility criteria for this study, all women selected were either using hormonal contraception or open to using hormonal contraception. In light of this, the trends discussed here may only apply to this subpopulation; hence, caution is advised when extrapolating these results to the wider female population.

\section{Acknowledgments}

The authors would like to thank Ogilvy 4D, Oxford, UK, for providing editorial assistance, and Bayer HealthCare AG for providing financial support. They would also like to thank Debra Corfield and Bridget McMahon from SKIM for providing assistance with the study design, implementation, and data analysis.

\section{Disclosure}

Johannes Bitzer has been a symposium speaker, advisory board member and received research grants and/or consulting fees from Bayer HealthCare AG, Amgen, Ferring, Lilly, MSD, Pfizer, Boehringer Ingelheim, Vifor and Warner Chilcott. Marco Serrani and Annalena Lahav are both employees of Bayer HealthCare AG. Editorial assistance was provided by Ogilvy 4D, Oxford, UK, and funded by Bayer HealthCare AG, Berlin, Germany. The authors report no other conflicts of interest in this work.

\section{References}

1. Fraser IS, Langham S, Uhl-Hochgraeber K. Health-related quality of life and economic burden of abnormal uterine bleeding. Expert Rev Obstet Gynecol. 2009;4(2):179-189.

2. Hallberg L, Högdahl AM, Nilsson L, Rybo G. Menstrual blood loss a population study. Variation at different ages and attempts to define normality. Acta Obstet Gynecol Scand. 1966;45(3):320-351. 
3. Matteson KA, Clark MA. Questioning our questions: do frequently asked questions adequately cover the aspects of women's lives most affected by abnormal uterine bleeding? Opinions of women with abnormal uterine bleeding participating in focus group discussions. Women Health. 2010;50(2):195-211.

4. Santer M, Wyke S, Warner P. What aspects of periods are most bothersome for women reporting heavy menstrual bleeding? Community survey and qualitative study. BMC Womens Health. 2007;7:8.

5. Shapley M, Jordan K, Croft PR. An epidemiological survey of symptoms of menstrual loss in the community. Br J Gen Pract. 2004; 54(502):359-363

6. Kadir RA, Aledort LM. Obstetrical and gynaecological bleeding: a common presenting symptom. Clin Lab Haematol. 2000;22(Suppl 1): S12-S16.

7. National Collaborating Centre for Women's and Children's Health. Heavy menstrual bleeding [homepage on the internet]. Dorchester: RCOG; 2007. Available from: http://www.nice.org.uk/nicemedia/ live/11002/30401/30401.pdf. Accessed August 13, 2012.

8. Coutinho EM, Segal SJ. Is Menstruation Obsolete? New York: Oxford University Press; 1999.

9. Grammaticos PC, Diamantis A. Useful known and unknown views of the father of modern medicine, Hippocrates and his teacher Democritus. Hell J Nucl Med. 2008;11(1):2-4.

10. O'Flynn N, Britten N. Menorrhagia in general practice - disease or illness. Soc Sci Med. 2000;50(5):651-661.

11. Janssen CA, Scholten PC, Heintz AP. Reconsidering menorrhagia in gynecological practice. Is a 30-year-old definition still valid? Eur J Obstet Gynecol Reprod Biol. 1998;78(1):69-72.

12. Santer M, Warner P, Wyke S. A Scottish postal survey suggested that the prevailing clinical preoccupation with heavy periods does not reflect the epidemiology of reported symptoms and problems. J Clin Epidemiol. 2005;58(11):1206-1210.
13. Cole SK, Billewicz WZ, Thomson AM. Sources of variation in menstrual blood loss. J Obstet Gynaecol Br Commonw. 1971;78(10):933-939.

14. Beaumont H, Augood C, Duckitt K, Lethaby A. Danazol for heavy menstrual bleeding [review]. Cochrane Database Syst Rev. 2007;3:CD001017.

15. Lethaby A, Augood C, Duckitt K, Farquhar C. Nonsteroidal antiinflammatory drugs for heavy menstrual bleeding [review]. Cochrane Database Syst Rev. 2007;4:CD000400.

16. Lethaby A, Farquhar C, Cooke I. Antifibrinolytics for heavy menstrual bleeding [review]. Cochrane Database Syst Rev. 2000;4:CD000249.

17. Lethaby A, Irvine G, Cameron I. Cyclical progestogens for heavy menstrual bleeding [review]. Cochrane Database Syst Rev. 2008;1:CD001016.

18. Lethaby AE, Cooke I, Rees M. Progesterone or progestogen-releasing intrauterine systems for heavy menstrual bleeding [review]. Cochrane Database Syst Rev. 2005;4:CD002126.

19. Fraser IS, McCarron G. Randomized trial of 2 hormonal and 2 prostaglandin-inhibiting agents in women with a complaint of menorrhagia. Aust N Z J Obstet Gynaecol. 1991;31(1):66-70.

20. Farquhar C, Brown J. Oral contraceptive pill for heavy menstrual bleeding [review]. Cochrane Database Syst Rev. 2009;4:CD000154.

21. Bayer HealthCare AG. Qlaira ${ }^{\circledR}$ EU summary of product characteristics [homepage on the internet]. Available from: http://db.cbg-meb.nl/mri/ spc/nlh-1230-001.pdf. Accessed August 13, 2012.

22. Fraser I, Parke S, Mellinger U, Machlitt A, Serrani M, Jensen J. Effective treatment of heavy and/or prolonged menstrual bleeding without organic cause: pooled analysis of two multinational, randomised, double-blind, placebo-controlled trials of oestradiol valerate and dienogest. Eur $J$ Contracept Reprod Health Care. 2011;16(4):258-269.
Open Access Journal of Contraception

\section{Publish your work in this journal}

Open Access Journal of Contraception is an international, peerreviewed, open access, online journal, publishing original research, reports, reviews and commentaries on all areas of contraception. In addition to clinical research, demographics and health-related aspects, the journal welcomes new findings in animal and preclinical studies

\section{Dovepress}

relating to understanding the biological mechanisms and practical development of new contraceptive agents. The manuscript management system is completely online and includes a very quick and fair peer-review system. Visit http://www.dovepress.com/testimonials.php to read real quotes from published authors. 